\title{
Serum magnesium levels and lung cancer risk: a meta-analysis
}

\author{
Xinghui Song ${ }^{1}$, Xiaoning Zhong ${ }^{1 *}$, Kaijiang Tang ${ }^{2}$, Gang $W^{3}$ and Yin Jiang ${ }^{2}$
}

\begin{abstract}
Background: Whether serum magnesium levels were lower in patients with lung cancer than that in healthy controls is controversial. The aim of this study was to identify and synthesize all citations evaluating the relationship between serum magnesium levels and lung cancer.

Methods: We searched PubMed, WanFang, China National Knowledge Internet (CNKI), and SinoMed databases for relevant studies before December 31, 2017. Two authors independently selected studies, extracted data, and assessed risk of bias.

Results: Eleven citations comprising 707 cases with lung cancer and 7595 healthy controls were included in our study. Serum magnesium levels were not significantly lower in patients with lung cancer [summary SMD $=0.193$, $95 \% \mathrm{Cl}=-1.504$ to 1.890$]$ when compared to health controls, with significant heterogeneity $\left(P^{2}=99.6 \%, P<0.001\right)$ found. Negative associations were found among Asian populations [summary SMD $=0.229,95 \% \mathrm{Cl}=-1.637$ to 2 . 094] and European populations [summary $\mathrm{SMD}=-0.168,95 \% \mathrm{Cl}=-0.482$ to 0.147 ]. No publication bias was found using the test of Egger and funnel plot.
\end{abstract}

Conclusions: Our study suggested that serum magnesium levels had no significant association on lung cancer risk.

Keywords: Magnesium level, Lung cancer, Meta-analysis, Healthy controls

\section{Background}

Lung cancer is the leading cause of death from cancer, resulting 1.38 million people deaths each year [1]. Its 5 -year survival rate is still as low as $15 \%$, and it is poor while compared with those in high incidence of other cancer [2]. Previous studies pointed out that lung cancer is the most common cancer among men and women, and both developed and developing countries bear a huge social and economic burden [3]. Previous publications proved that both genetic and environment factors were related to lung cancer risk [4-7]. Furthermore, trace-heavy elements also played a significant role on human health and disease [8, 9], as well as lung cancer [10].

Magnesium is one of the trace elements in our bodies, and to date, some papers had been published to investigate the association between serum magnesium levels and lung cancer risks. Two papers [11, 12] reported a higher of serum magnesium level in cases with lung

\footnotetext{
* Correspondence: xnzhong101@sina.com

1 Department of respiration, the First Affiliated Hospital of Guangxi Medical

University, N0.6 Shuangyong Road, Nanning 530021, Guangxi, China

Full list of author information is available at the end of the article
}

cancer, while six papers [13-18] found a lack of significant association. Conversely, three papers [19-21] suggested that it is lower in lung cancer cases when compared to the healthy controls. Therefore, the aim of this study was to identify and synthesize all citations evaluating the relationship between serum magnesium levels and lung cancer risk.

\section{Methods}

Study selection

A comprehensive literature search was conducted in platforms of PubMed, WanFang, China National Knowledge Internet (CNKI), and SinoMed databases up to December 31, 2017. Free words adopted were as follows: "magnesium" or "Mg" combined with "lung cancer" or "lung carcinoma" without restrictions. Reference lists of the studies retrieved were also examined to find any additional study potentially unidentified. The course of study selection was completed by two investigators independently. Any resulting discrepancies were resolved by a third reviewer. 
The inclusion criteria were as follows: (i) having a prospective design or a case-control design or a cross-sectional study; (ii) evaluating the association between serum magnesium levels and risk of lung cancer; (iii) reporting mean and standard deviation (SD) of magnesium levels (or sufficient data to compute them) both in lung cancer patients and healthy controls; and (iv) studies published in English language or Chinese language. If more than one article referred to the same populations, only the study that included the most lung cancer cases or the latest publication was included.

\section{Data extraction and quality assessment of studies}

Two investigators independently extracted the following data: (1) first author's last name; (2) publication year; (3) study design; (4) country; (5) number of lung cancer cases and participants; (6) sex of cases; (7) age range or mean age of the cases; (8) mean and SD of magnesium levels both in lung cancer patients and healthy controls; and (9) method used for detection of magnesium. Any resulting discrepancies were resolved by a third reviewer.

The methodological quality of studies was evaluated independently by two researchers using the NewcastleOttawa Quality Assessment Scale [22]. The three components were as follows: (1) patient selection (4 points); (2) comparability (2 points); and (3) outcome (3 points) for a total score of 9 points.

\section{Statistical analysis}

Standardized mean difference (SMD) and their 95\% confidence interval (CI) were calculated for relationship between serum magnesium levels and lung cancer risk. A random effect model was used in our meta-analysis [23]. The heterogeneity among studies was evaluated with $I^{2}$ and Q tests. [24]. $P<0.05$ in Q test and $I^{2}>50 \%$ indicated statistically significant heterogeneity [25]. Metaregression was adopted to assess the between-study heterogeneity. Egger's regression asymmetry test [26] and funnel plot [27] were used to visually examine publication bias on study outcome. Statistical analyses were performed using STATA version 12.0 (Stata Corporation, College Station, TX, USA). A two-sided $P<0.05$ was defined as statistical significance.

\section{Results}

\section{Study characteristics}

As shown in Fig. 1, the initial 486 articles screened through databases of PubMed, WanFang, China National Knowledge Internet (CNKI), and SinoMed databases searching and 1 additional record identified through other sources. There are 372 articles that were reviewed in the title and abstract while excluding the duplications from different databases. Three hundred and forty two of
372 articles were excepted when screened on the basis of title and abstract; 30 articles were examining full texts. Nineteen studies were further excluded (reviews, not report mean or SD, animal studies, letter to the editors). Finally, 11 articles [11-21] were eligible to be included in the analysis comprising 707 patients with lung cancer and 7595 healthy controls. All the included studies were case-control studies. Nine studies were carried out from China, 1 from Spain, and 1 from Turkey. Ten of the included studies used the methods of atomic absorption spectrophotometer measurements for detection of magnesium. In the study quality assessment, all the included studies were with a score greater or equal to 6 . The basic features of all citations are shown in Table 1.

\section{Serum magnesium levels and lung cancer risk}

Pooled results suggested that magnesium levels in patients with lung cancer was not significantly lower than healthy controls [summary SMD $=0.193,95 \% \mathrm{CI}=-1.504$ to $1.890, I^{2}=99.6 \%, P_{\text {for heterogeneity }}<0.001$ ] (Fig. 2). When we performed the subgroup analysis by geographic location, the association was not significant either in Asian populations [summary SMD $=0.229,95 \% \mathrm{CI}=-1.637$ to 2.094] or in European populations [summary SMD = $-0.168,95 \% \mathrm{CI}=-0.482$ to 0.147$]$.

\section{Sources of heterogeneity and meta-regression}

Meanwhile, $I^{2}$ was $99.6 \%(p<0.001)$ for the pooled sensitivity, suggesting high heterogeneity in the sample of studies. Univariate meta-regression was then carried out to determine the reason of heterogeneity. However, there were no significant contributions about publication year, case number, geographic location, sex, and different methods on this high between-study heterogeneity.

\section{Sensitivity analysis and publication bias}

Sensitivity analysis conducted while removing one study at the time revealed that no single study had essential effect on the whole result. Figure 3 showed that no publication was considered by the funnel plot method on the basis of data, as well as the Egger's test $(P=0.586)$.

\section{Discussion}

In this study, we assessed the association between serum magnesium levels and risk of lung cancer. We did not find a positive association between serum magnesium levels and lung cancer risk. Through our subgroup analysis, we further found no significant association among Asian and European populations. Significant heterogeneity between studies observed in this meta-analysis should be considered as a major limitation of these findings; however, heterogeneity was mainly related to strength of the association rather than the direction of risk estimate, 


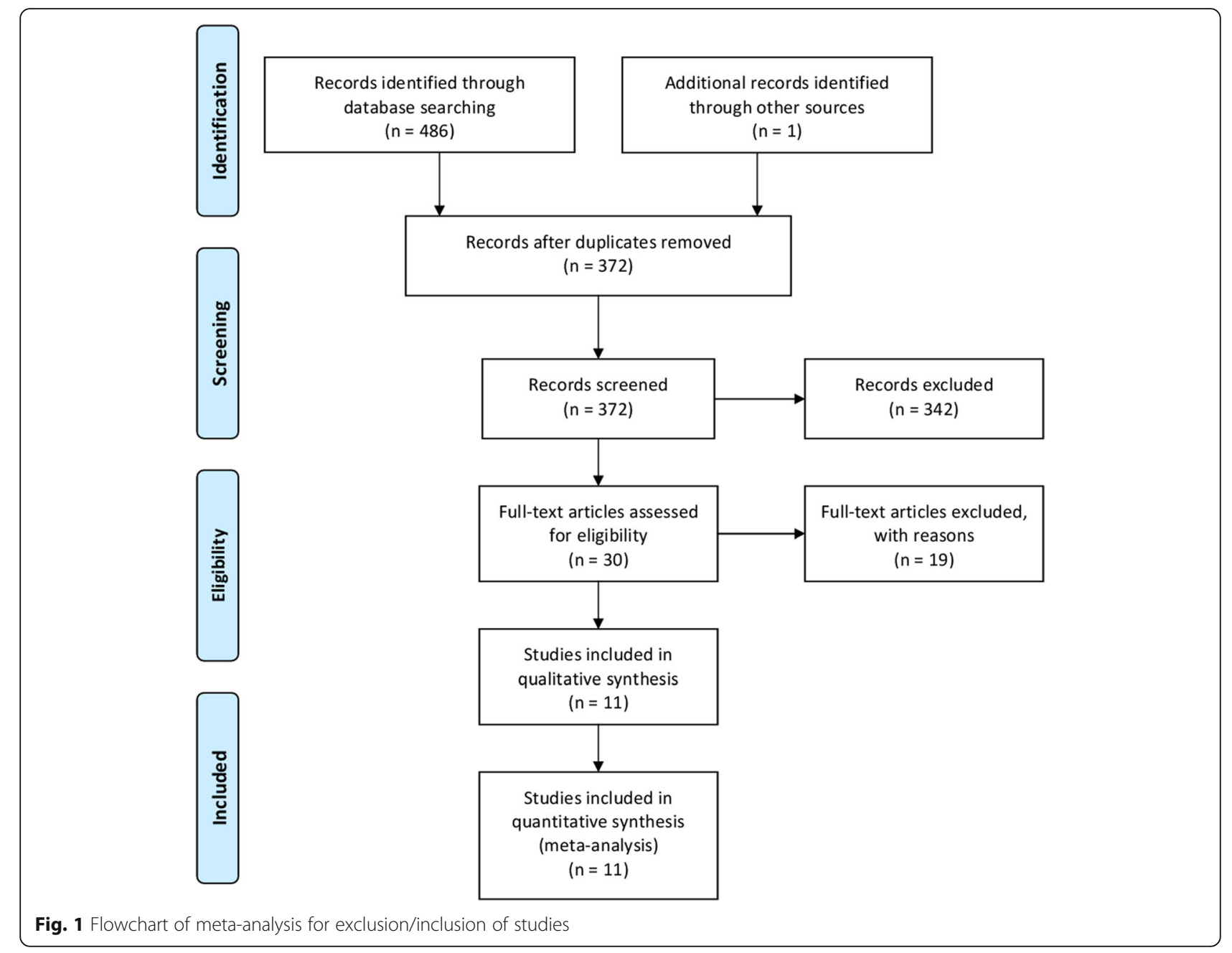

suggesting overall promising findings on the outcome investigated in the present study.

Two previous prospective cohort studies concluded that higher category of dietary magnesium intake had no significant association on lung cancer risk among German population and China population [28, 29]. However, a report [30] had been resulting that higher magnesium levels in drinking water could reduce the risk of lung cancer deaths in women. To our knowledge, no comprehensive analysis had been published to assess the serum magnesium levels on lung cancer risk. In our study, we did not find significant association of lower serum magnesium levels in patient with lung cancer. However, level of magnesium in other disease may be in the normal range, and that magnesium can have an effect on this disease [31].

The existence of heterogeneity among the studies, which is common in meta-analyses [32], may affect the pooled results. Meta-regression was performed to find the potential covariates (publication year, case number, geographic location, sex, and different methods to detect magnesium levels) which may cause this high heterogeneity. However, no covariate was found to significantly contribute to heterogeneity. In our study, most of the included studies obtained nonsignificant association between serum magnesium levels and lung cancer risk. Only one study [12] reported that serum magnesium level in patient with lung cancer is extremely higher than that in healthy controls. We reviewed the article again and confirmed the data exacted from the study; no error was made. Sensitivity analysis was performed, and no study had essential effect to the significant between-study heterogeneity and the whole result. On the other hand, we used a random effect model to combine the results. As we all know, random effect model had wider rage about $95 \%$ CI than fix effect model and could obtain more accurate results. Furthermore, only three studies $[12,13,17]$ reported the types and staging of lung cancer, which may also be a factor on the between-study heterogeneity. Therefore, studies with detailed information of types and staging of lung cancer are wanted to further explore this association. 


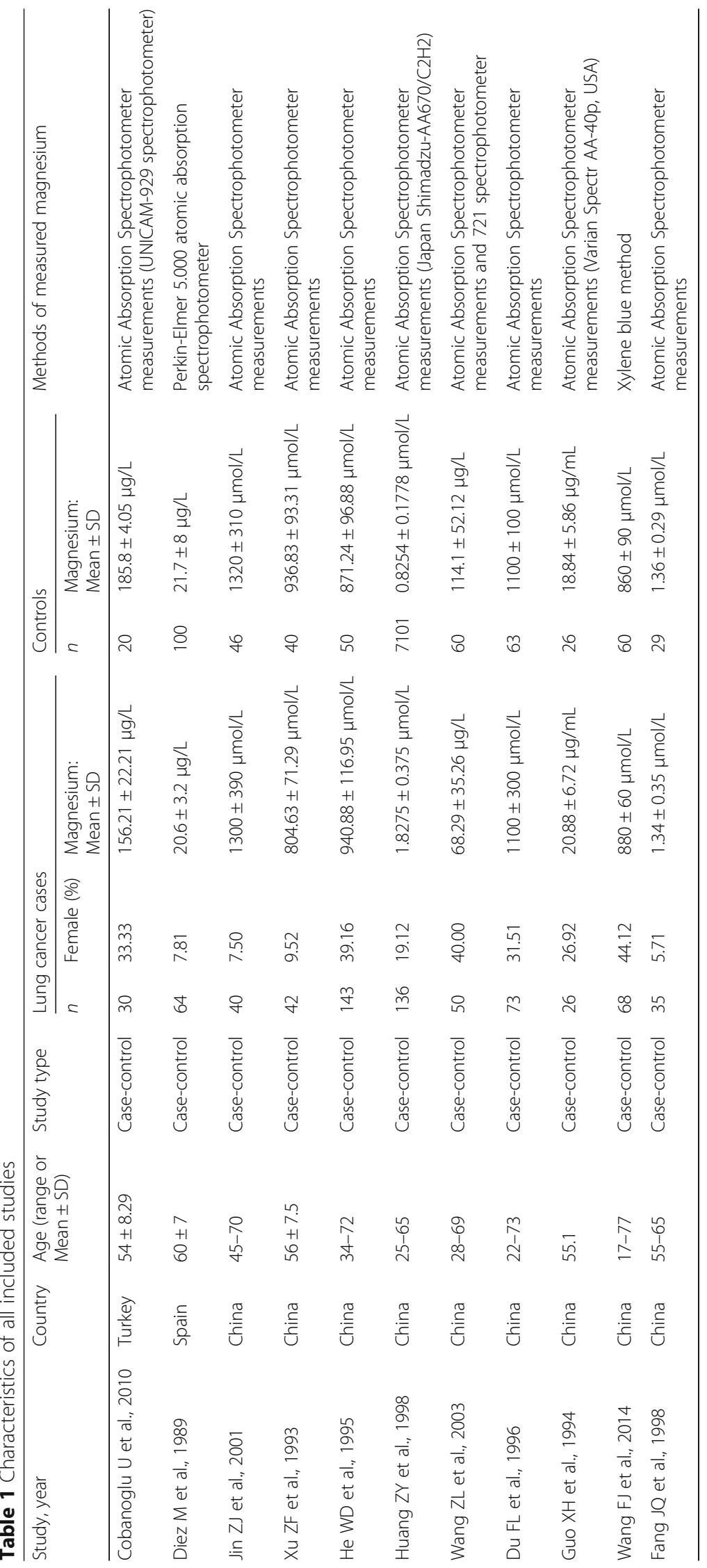




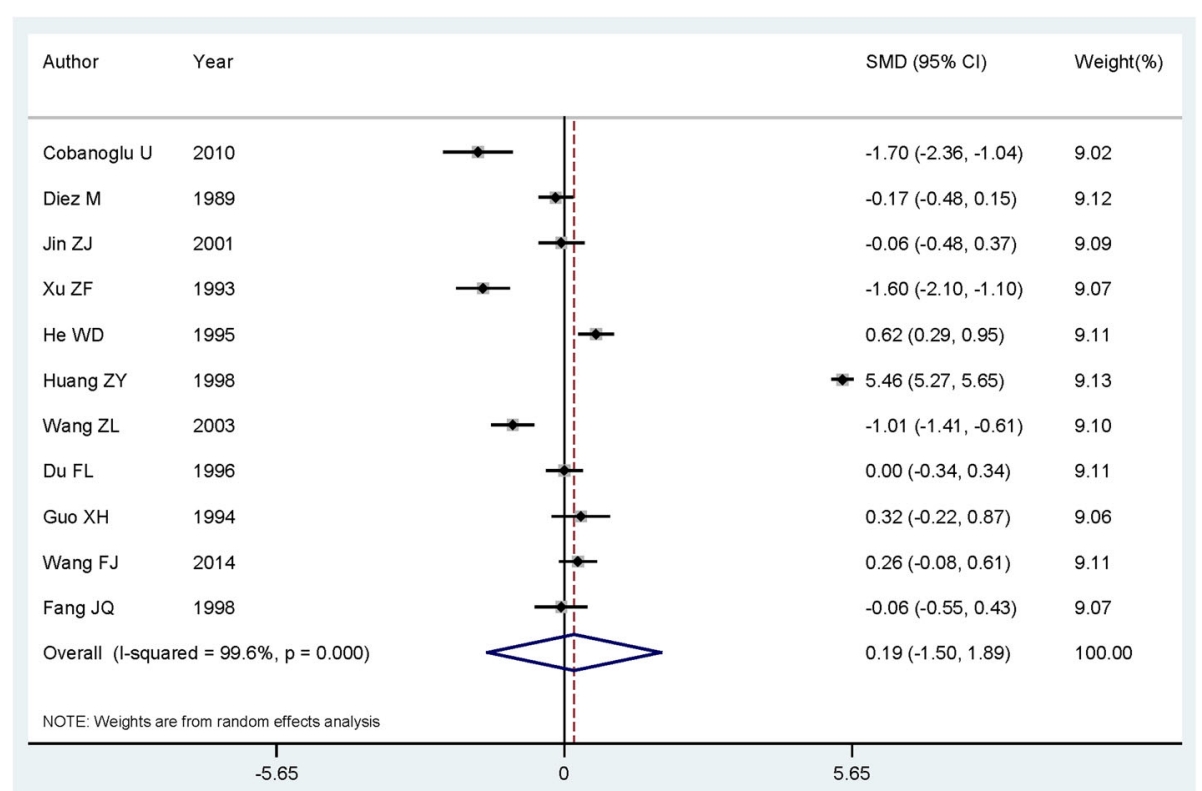

Fig. 2 The forest plot of the relationship between serum magnesium levels and lung cancer risk

Some advantages existed in our study. Firstly, a comprehensive literature search was performed to investigate the relationship between serum magnesium levels and lung cancer risk. Secondly, most of the included studies involved large numbers of patients and healthy controls, and this may strengthen the power of the pooled results. Thirdly, there was no significant publication when tested by Egger and funnel plot, which indicates that our results are stable.

The present study has some limitations. Firstly, the individual studies may have failed to control for potential confounders, which may introduce bias in an unpredictable direction. Secondly, ten of 11 studies were from Asia, and 9 were from China, and thus, more related researches from other countries are wanted to verify the association between geographic location and lung cancer risk.

\section{Conclusions}

Based on the obtained results, we concluded that serum magnesium levels may have no significant association in patients with lung cancer. As we experienced some limitations in our study, such as more studies were from Asia, further studies are wanted to confirm this finding.

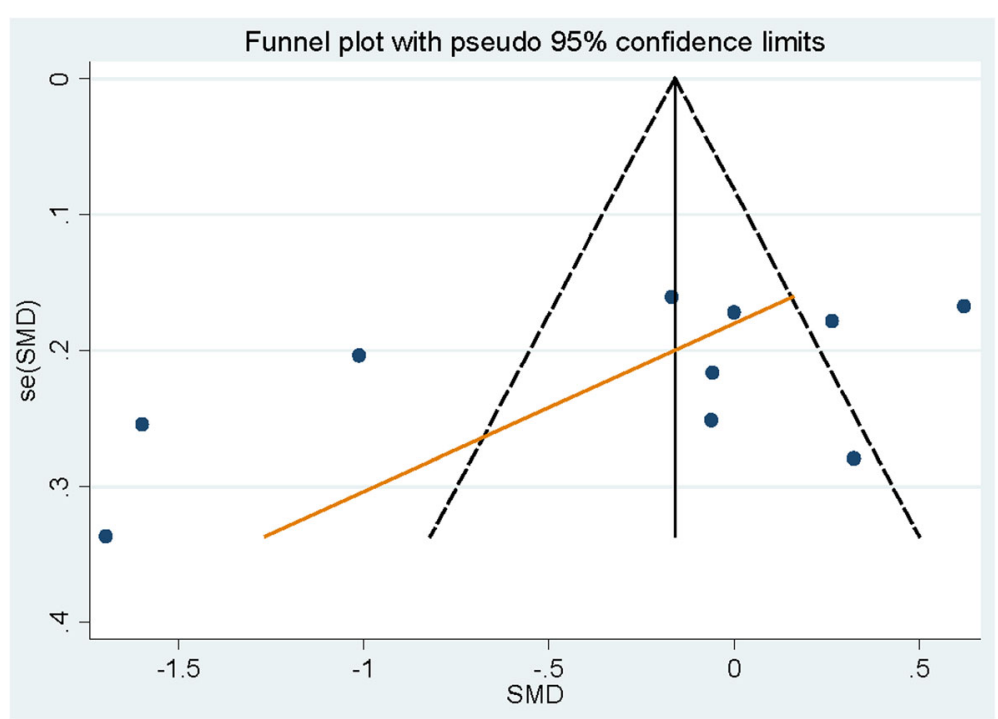

Fig. 3 Funnel plot for the analysis of publication bias between serum magnesium levels and lung cancer risk 


\section{Abbreviations}

Cl: Confidence intervals; SD: Standard deviation; SMD: Standard mean differences

\section{Availability of data and materials}

Data sharing is not applicable to this article as no datasets were generated or analyzed during the current study.

\section{Authors' contributions}

XNZ was the guarantor of integrity of the entire study and was responsible for the definition of intellectual content and manuscript editing. XHS contributed to the study concepts, study design, and data analysis and was responsible for the manuscript preparation. KJT and GW were responsible for the literature research. GW carried out the experimental studies. KJT and YJ were responsible for the data acquisition. All authors read and approved the final manuscript.

\section{Competing interest}

The authors declare that they have no competing interests.

\section{Ethics approval and consent to participate}

Not applicable.

\section{Consent for publication}

Not applicable.

\section{Publisher's Note}

Springer Nature remains neutral with regard to jurisdictional claims in published maps and institutional affiliations.

\section{Author details}

${ }^{1}$ Department of respiration, the First Affiliated Hospital of Guangxi Medical University, N0.6 Shuangyong Road, Nanning 530021, Guangxi, China. 2Department of rheumatism, Liuzhou Worker's Hospital, Liuzhou 545005, Guangxi, China. ${ }^{3}$ Department of neurosurgery, Liuzhou General Hospital, Liuzhou 545006, Guangxi, China.

Received: 25 January 2018 Accepted: 5 July 2018

Published online: 12 July 2018

\section{References}

1. Torre LA, Bray F, Siegel RL, et al. Global cancer statistics, 2012. CA Cancer J Clin. 2015;65(2):87-108

2. Tsao AS, Scagliotti GV, Bunn PA Jr, et al. Scientific advances in lung cancer 2015. J Thorac Oncol. 2016;11(5):613-38

3. Minguet J, Smith KH, Bramlage P. Targeted therapies for treatment of non-small cell lung cancer-recent advances and future perspectives. Int $J$ Cancer. 2016;138(11):2549-61.

4. Liu C, Cui H, Gu D, et al. Genetic polymorphisms and lung cancer risk: evidence from meta-analyses and genome-wide association studies. Lung Cancer. 2017;113:18-29.

5. Wang J, Liu Q, Yuan S, et al. Genetic predisposition to lung cancer: comprehensive literature integration, meta-analysis, and multiple evidence assessment of candidate-gene association studies. Sci Rep. 2017;7(1):8371.

6. Papadopoulos D, Papadoudis A, Kiagia M, et al. Nonpharmacologic interventions for improving sleep disturbances in patients with lung Cancer: a systematic review and meta-analysis. J Pain Symptom Manag. 2018;55(5): 1364-81.

7. Poinen-Rughooputh S, Rughooputh MS, Guo Y, et al. Occupational exposure to silica dust and risk of lung cancer: an updated meta-analysis of epidemiological studies. BMC Public Health. 2016;16(1):1137.

8. Demir N, Basaranoglu M, Huyut Z, et al. The relationship between mother and infant plasma trace element and heavy metal levels and the risk of neural tube defect in infants. J Matern Fetal Neonatal Med. 2017. Epub ahead of print.

9. Wu T, Bi X, Li Z, et al. Contaminations, sources, and health risks of trace metal (loid) $s$ in street dust of a Small City impacted by artisanal Zn smelting activities. Int J Environ Res Public Health. 2017;14(9):961.

10. Zablocka-Slowinska K, Placzkowska S, Prescha A, et al. Serum and whole blood $\mathrm{Zn}, \mathrm{Cu}$ and $\mathrm{Mn}$ profiles and their relation to redox status in lung cancer patients. J Trace Elem Med Biol. 2018;45:78-84.
11. He WD. Detection and analysis of some trace elements in serum of patients with lung cancer. Jiujiang Med J. 1995:10(2):69-71.

12. Huang ZY, Hu FD. Comparative study of serum trace elements in patients with lung cancer. Shanxi Clin Med J. 1998;17(2):114-6.

13. Diez M, Arroyo M, Cerdan FJ, et al. Serum and tissue trace metal levels in lung cancer. Oncology. 1989:46(4):230-4.

14. Jin ZJ, Qian LQ, Dong GQ, et al. Measurement and analysis of serum copper zinc and magnesium in patients with lung cancer and gastric cancer. Shaanxi Med J. 2001;30(3):165-6.

15. Du FL, Li ZM, Cao MJ, et al. Determination of serum copper, zinc, magnesium and iron in patients with pulmonary tuberculosis, chronic bronchitis, pulmonary heart disease and lung cancer. J of Xi'an Med University. 1996;17(3):348-50.

16. Guo XH, Li PF, Peng FK, et al. Relationship between serum zinc, copper, manganese and lung cancer. Chin Pub Heal. 1994;10(4):156-7.

17. Wang FJ, Zhao ZE, Wen JB, et al. Levels of some major elements in patients with lung cancer: a retrospective analysis. Chin J Gen Pract. 2014;12(4):528-30.

18. Fang JQ, Mei YL, Li LJ, et al. Analysis of selenium, copper, magnesium, magnesium and selenium contents in elderly patients with lung cancer Ningxia Med J. 1998;20(4):247-8.

19. Cobanoglu U, Demir H, Sayir F, et al. Some mineral, trace element and heavy metal concentrations in lung cancer. Asian Pacific journal of cancer prevention : APJCP. 2010;11(5):1383-8

20. Xu ZF, Sun YC, Zhang CW, et al. Clinical significance of changes of serum copper, zinc and magnesium contents in patients with lung cancer. J of Second Mil Med Univ. 1993;14(2):195-6.

21. Wang ZL, Zhang W, Zhang HY, et al. Determinat ion of serum trace elements and their clinical value in patients with lung cancer. Clin Focus. 2003;18(4):183-5.

22. Stang A. Critical evaluation of the Newcastle-Ottawa scale for the assessment of the quality of nonrandomized studies in meta-analyses. Eur J Epidemiol. 2010;25(9):603-5.

23. DerSimonian R, Laird N. Meta-analysis in clinical trials. Control Clin Trials. 1986;7(3):177-88.

24. Higgins JP, Thompson SG, Deeks JJ, et al. Measuring inconsistency in metaanalyses. BMJ. 2003;327(7414):557-60.

25. Higgins JP, Thompson SG. Controlling the risk of spurious findings from meta-regression. Stat Med. 2004;23(11):1663-82.

26. Egger M, Davey Smith G, Schneider M, et al. Bias in meta-analysis detected by a simple, graphical test. BMJ. 1997;315(7109):629-34.

27. Begg CB, Mazumdar M. Operating characteristics of a rank correlation test for publication bias. Biometrics. 1994:50(4):1088-101.

28. Li K, Kaaks R, Linseisen J, et al. Dietary calcium and magnesium intake in relation to cancer incidence and mortality in a German prospective cohort (EPIC-Heidelberg). Cancer Causes Control. 2011;22(10):1375-82.

29. Takata Y, Shu XO, Yang G, et al. Calcium intake and lung cancer risk among female nonsmokers: a report from the Shanghai Women's Health Study. Cancer Epidemiol Biomarkers Prev. 2013;22(1):50-7.

30. Cheng MH, Chiu HF, Tsai SS, et al. Calcium and magnesium in drinkingwater and risk of death from lung cancer in women. Magnes Res. 2012; 25(3):112-9.

31. Srebro DP, Vučković SM, Dožić IS, et al. Magnesium sulfate reduces formalin-induced orofacial pain in rats with normal magnesium serum levels. Pharmacol Rep. 2018;70(1):81-6.

32. Munafo MR, Flint J. Meta-analysis of genetic association studies. Trends Genet. 2004;20(9):439-44.

Ready to submit your research? Choose BMC and benefit from

- fast, convenient online submission

- thorough peer review by experienced researchers in your field

- rapid publication on acceptance

- support for research data, including large and complex data types

- gold Open Access which fosters wider collaboration and increased citations

- maximum visibility for your research: over $100 \mathrm{M}$ website views per year

\section{At BMC, research is always in progress.}

Learn more biomedcentral.com/submissions 